Classification

Physics Abstracts

61.16Di - 61.70Im - 61.70Wp

\title{
CBED strain measurements in boron implanted silicon
}

\author{
Roberto Balboni and Stefano Frabboni \\ Dipartimento di Fisica, Universita' di Modena, Via G. Campi 213/A, I-41100, Modena, Italy \\ (Received December 05, 1991; accepted February 10, 1992)
}

\begin{abstract}
Strain measurements were performed by convergent-beam electron diffraction on both plan-view sample and cross-sections of silicon wafers, boron implanted at liquid nitrogen temperature. In the plan-view specimens, the strain value measured in the boron-doped layer agrees with previous $\mathrm{X}$-ray double crystal diffraction analyses. In the cross-sectioned specimens, a profile of the strain was obtained, showing a difference between the boron-doped layer and the end-of-range, interstitial-rich layer. In the latter samples the quantitative agreement with X-ray measurements is reached when extra-relaxation along the thinning direction is taken into account.
\end{abstract}

\section{Introduction.}

Among the experimental techniques available for the structural characterisation of electronic materials, transmission electron microscopy (TEM) is particularly potent, as it allows a variety of spectroscopic, imaging and diffraction information to be obtained from a single microarea of the sample. Typical problems solvable by TEM are: type, density and depth distribution of defects, detection of second phases, microchemical analyses etc.. Moreover, since 1977 [1], convergent beam electron diffraction (CBED) has extended the spectrum of structural information available from TEM analyses to the study of strain in the range of $10^{-4}-10^{-3}$, the sensitivity of this technique being one order of magnitude higher than that of conventional selected area diffraction.

Recently, CBED has found an increasing number of applications for electronic materials thanks to the increasing interest in understanding the accommodation of misfit strains during the epitaxial growth processes, and due to the dependence of electron band structure on strain. In semiconductor heterostructures and superlattice technology, where "strain engineering" has provided a mean of controlling electrical properties, CBED techniques have been applied to the study of strains or rigid shifts across the epitaxial interfaces [2-5].

Similarly, the capabilities of CBED techniques in detecting and measuring strain fields are also of potential interest in ion implanted materials, although, to the best of our knowledge, no work has yet been reported on this subject. In fact, it is known that, in these specimens, the implanted dopant atoms, which are located in substitutional sites after annealing, produce a strain field generated by the difference in tetrahedral radius between themselves and the host silicon atoms [6]. 
This strain, which is, respectively, compressive for oversized and tensile for undersized dopants, is strongly anisotropic because of the small thickness of the implanted layers with respect to that of the silicon wafers. It can be described by the so-called tetragonal strain, $\varepsilon_{\mathrm{T}}$, if the deformation values do not exceed the critical stress for generation of misfit dislocations. The tetragonal strain is defined as the ratio of the lattice parameter variation perpendicular to the surface to the unstrained lattice constant in the plane parallel to the surface of the wafer. It is equal to the natural mismatch, $f$, multiplied by a suitable factor equal to $(1+\nu) /(1-\nu)$ (here $\nu$ is the Poisson ratio) according to the anisotropic elasticity theory [7]. By measuring this strain, it should be possible to obtain an evaluation of the concentration of dopant atoms in substitutional sites, assuming a linear strain-versus-concentration behaviour, and that coherent particles such as microprecipitates are not present. Like dopant atoms in substitutional sites, interstitial or vacancy-type microdefects also produce an anisotropic strain field (compressive or tensile) induced by the difference in volume between the perfect and the defective crystal [8]. Discriminating by CBED between the two kinds of strain, should permit determination of microdefect type.

In the following study, CBED techniques are applied to the analysis of silicon wafers implanted with boron at liquid nitrogen temperature in order to measure the tetragonal strain in the surface layers of monocrystalline doped film, $\mathrm{Si}(\mathrm{B})$. Measurements are performed both in cross-section and in plan-view thinned samples. The detection of the strain field generated by small defects located at the end of the ion range is also presented, and the results obtained are discussed and compared with previous X-ray double crystal diffraction (DCD) analyses [9].

\section{Experimental.}

Chemo-mechanically polished n-type, phosphorus doped, 6 inch [001] oriented silicon wafers were implanted with ${ }^{11} \mathrm{~B}$ ions with a low current, low energy Extrion implanter, at a fluence of $7 \times 10^{15}$ ions $/ \mathrm{cm}^{2}$ with an energy $30 \mathrm{keV}$. The samples were kept at liquid nitrogen temperature during the implantation in order to produce a boron doped amorphous silicon layer. The thermal treatment was performed in vacuum at $500^{\circ} \mathrm{C}$ for $30 \mathrm{~min}$.

Plan-view and [110] cross-section TEM specimens were prepared from both as-implanted and annealed samples. Plan-view specimens were thinned by chemical etching $\left(82 \% \mathrm{HNO}_{3}+18 \%\right.$ $\mathrm{HF}$ ). Cross sections were prepared using a previously reported technique [10] consisting of gluing, sawing, mechanical lapping and ion milling. The ion milling was performed with a Gatan Duomill, at low current, low voltage $(4 \mathrm{kV})$ and at an incidence angle of $14^{\circ}$ in order to reduce thinning artefacts.

Conventional TEM observation, CBED and Large Angle CBED (LACBED) analyses were performed with a Philips CM30 equipped with a Gatan liquid-nitrogen double-tilt specimen holder for High Order Laue Zone (HOLZ) line strain measurements (the specimen temperature was about $100 \mathrm{~K}$ during the observation). Although the used spot size was very small (about $7 \mathrm{~nm}$ ), the evaluated spatial resolution was about $20 \mathrm{~nm}$ because of the beam broadening and of the spherical aberration of the illumination system. LACBED patterns were taken at $300 \mathrm{kV}$ with a $200 \mu \mathrm{m}$ second condenser aperture, using a $10 \mu \mathrm{m}$ selected area aperture in order to select a single diffracted beam.

DCD measurements were performed on the annealed samples only. The experimental set-up of DCD measurements is extensively described elsewhere [11]. 


\section{The CBED (and LACBED) technique.}

The methods of CBED strain measurements have been extensively reviewed by Humphreys et al. [12] and Cherns and Preston [13] and their application to the detection and measurements of the tetragonal strain will only be summarized here.

HOLZ line method. - In the bright field disc of a CBED zone axis pattern an intersecting set of very narrow HOLZ deficiency lines is often present. The position of these lines is sensitive, among other parameters, to small changes in lattice constants and microscope voltage. In order to quantify the unknown lattice parameter variations between the strained implanted layers and the silicon substrate, it is first necessary to match the perfect silicon experimental pattern with a kinematically simulated one taking the acceleration voltage as a fitting parameter. Then, assuming the transferability of this value from the silicon to the strained layers, the unknown lattice parameter variations are determined by fitting the experimental pattern with the calculated one, taking the lattice constants as fitting parameters. This method has been extensively discussed by Lin et al. [14] and Bithell and Stobbs [15]. These authors have shown that the above assumptions, although physically unrealistic, are nevertheless valid for comparison between materials with differences in mean atomic number lower than about 5 units. The transferability assumption should therefore be valid in our case as the difference in mean atomic number between $\mathrm{Si}(\mathrm{B})$ and $\mathrm{Si}$ is about 0.05 units, the boron concentration being lower than 0.6 atomic percent, which is the limit for boron precipitation in silicon during the solid phase epitaxial process [16].

The HOLZ line method is particularly suitable in cross-sectioned samples, since the interface between the substrate and the ion implanted layer is parallel to the viewing direction, and so distinct HOLZ line patterns from the substrate and different areas of the strained layers can be independently recorded.

Bragg contour method in large angle CBED (LACBED) patterns. - The distortion in the cubic lattice caused by tetragonal strain, leads to a rotation of the planes inclined at a particular angle with respect to the surface. This is schematically shown in figure 1 , where a layer with a tensile strain is present: the effect on a plane (say an (084) plane) forming an angle $\Theta$ with the surface, is to tilt it away from the [001] direction. The amount of rotation is related to the tetragonal strain, $\varepsilon_{\mathrm{T}}$, with the relation:

$$
2 \Delta \Theta=\varepsilon_{\mathrm{T}} \sin (2 \Theta)
$$

The tetragonal strain can therefore be readily obtained by measuring $\Delta \Theta$. This can be achieved by means of the LACBED technique in a plan-view specimen, where both the unperturbed and tilted planes may be observed in their Bragg positions owing to the large illumination angle used, the convergence of which is only limited by the size of the largest $C_{2}$ aperture available (in our case $200 \mu \mathrm{m})$. Thus a rocking curve is obtained for each plane, and Bragg contour deficiency lines are visible in the transmitted beam; if a strained layer is present, medium and high index reflections show a split central peak. $\Delta \Theta$ can therefore be directly measured from the diffraction pattern.

Experimentally, these patterns are obtained by bringing the incident beam to a focus below the specimen and/or by raising the specimen from the eucentric position so that the focused spots due to the transmitted and diffracted beams are spatially separated in the image plane of the objective lens; a selected area aperture can then be used to select a single beam. The extended rocking curves for the chosen reflections are observable with a superimposed defocused specimen image by switching the microscope to the diffraction mode[17]. 


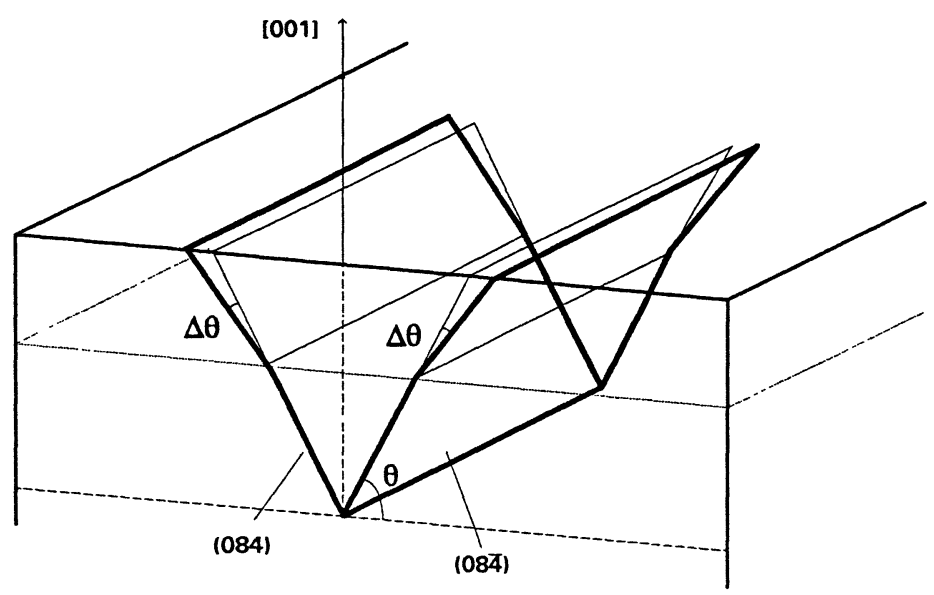

Fig. 1. - A plane in the strained layer is tilted of an angle $\Delta \Theta$ with respect to its direction in the substrate. The tilt angle for two $\{840\}$ planes is shown if a tensile strain is assumed.

\section{Results and discussion.}

4.1 MORPHOLOGY. - As reported in a previous paper [18], the as-implanted specimen shows a superficial amorphous layer $155 \mathrm{~nm}$ thick.

Figure 2 shows a [110] cross-sectional TEM image of the sample annealed at $500^{\circ} \mathrm{C}$ for $30 \mathrm{mn}$, where the low temperature thermal treatment has caused the epitaxial regrowth of the crystal. In fact, a boron-doped, monocrystalline, silicon film, Si(B), free of extended defects may be seen to extend from the surface down to a depth of about $155 \mathrm{~nm}$ (corresponding to the original amorphous crystalline interface). The absence of extended defects in the regrown layer was also checked in the plan-view sample, suggesting a defect density lower than $10^{+6} \mathrm{~cm}^{-2}$. Beneath this crystalline film a thin band of "end of range" microdefects is visible. This morphological picture is consistent with the DCD measurements reported in figure 3. Using this technique, a tensile strain is detected in the case where the surface region is believed to contain substitutional boron atoms. However, in the case of interstitial-type defects with a low density of microdefects (as revealed by TEM), a positive (i.e. compressive) strain is present [11].

4.2 CBED MEASUREMENTS. - CBED analyses were performed on both cross-sectioned and plan-view samples. The experimental results of CBED and LACBED analyses are summarised in figures 4 and 6 and in table $I$.

Figures 4a,b,c show the experimental [310] zone axis HOLZ line patterns relative to the probed points P1, P2, P3 (see Fig. 2) of the cross-sectioned samples. The diffraction patterns from points $\mathrm{P} 2$ and $\mathrm{P} 3$ behave differently with respect to the reference state (Fig. 4a). In particular the segment $\mathrm{ab}$ in figure $4 \mathrm{a}$ is longer than that in figure $4 \mathrm{~b}$, but shorter than that of figure $4 \mathrm{c}$. In order to obtain significant information about the strain values at points $\mathrm{P} 2$ and $\mathrm{P} 3$, we have performed kinematical simulations of HOLZ line patterns using the HL routine of the EMS program by P.A. Stadelmann [19]. By comparing the computed diffraction patterns of figures $4 \mathrm{~d}, \mathrm{e}, \mathrm{f}$ with the experimental ones it is possible to deduce that the point $\mathrm{P} 2$ has a positive strain. This compressive strain, generated by interstitial type defects, is difficult to measure because of the marked broadening of the HOLZ lines (probably due to the static disorder). It causes unacceptable un- 


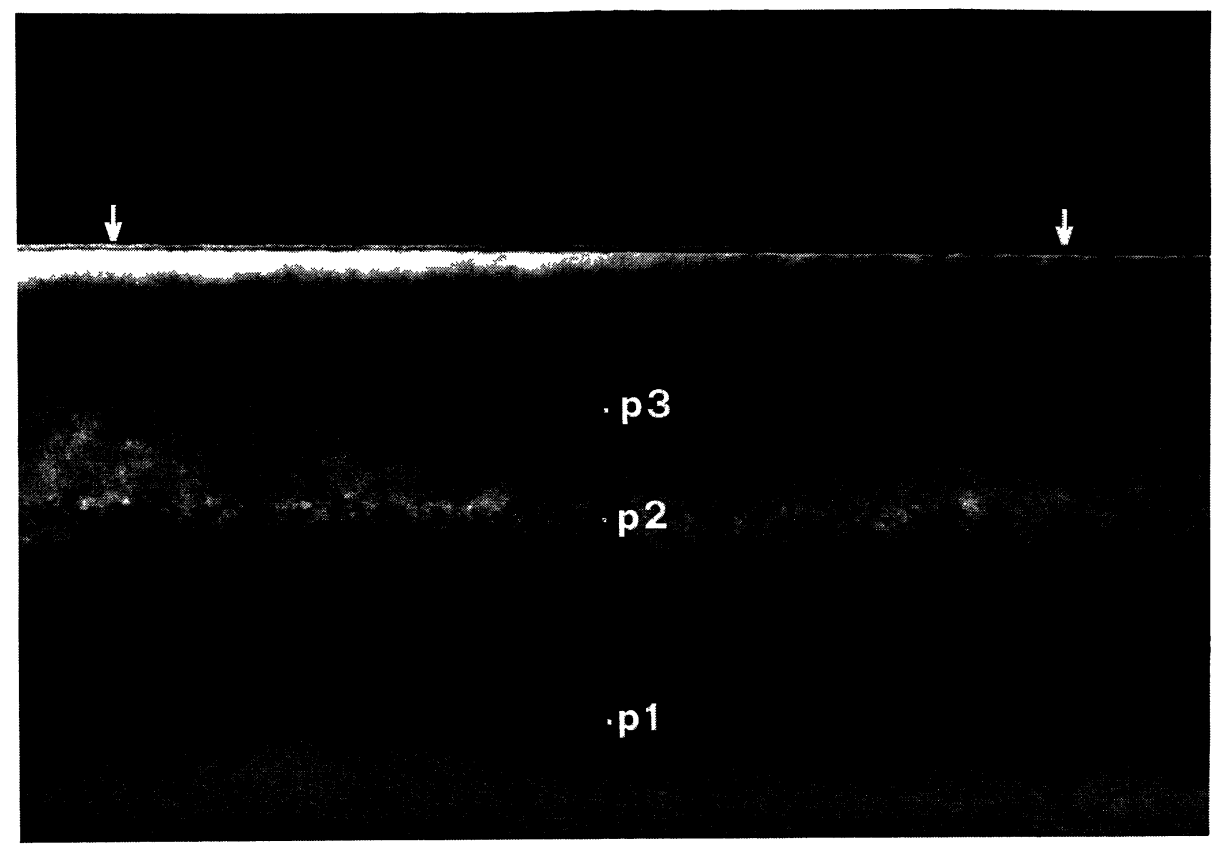

Fig. 2. $-[110]$ cross section of the sample annealed at $500^{\circ} \mathrm{C}$ for $30 \mathrm{mn}$. Dark field, $g=(111)$. The arrows mark the surface of the specimen. P1, P2 and P3 indicate the areas where the CBED patterns have been taken.

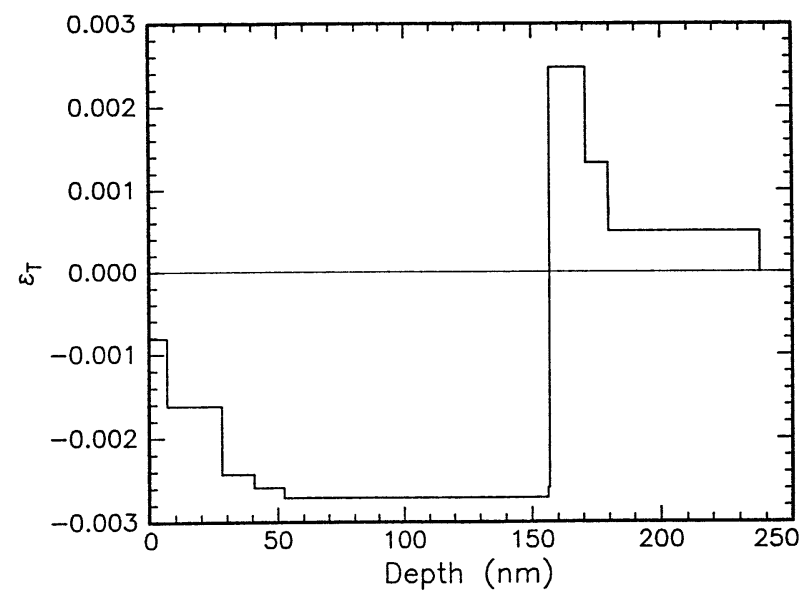

Fig. 3. - X-ray DCD strain depth profile.

certainties in the lattice parameter measurements. Instead, P3 has a negative (i.e. tensile) strain, produced by substitutional boron atoms. In the latter case, in order to fit the experimental patterns with the calculated ones, it was necessary to change all the lattice parameters and the angle $\gamma$ between the [100] and the [010] direction (see Tab. I). These adjustements, which are different from those expected in the absence of thinning are the consequence of the additional relaxation 
Table I. - Comparison between strain values measured by CBED in cross-sectioned specimens $\left(\varepsilon_{\mathrm{T}}^{\mathrm{CBED}}\right)$ and by LACBED in plan-view specimens $\left(\varepsilon_{\mathrm{T}}^{\mathrm{T}}\right)$ with $D C D\left(\varepsilon_{\mathrm{T}}^{\mathrm{DCD}}\right)$ and "fully relaxed" strain values $\left(\varepsilon^{\prime}\right)$.

\begin{tabular}{|c|c|c|c|c|c|c|c|c|}
\hline & $a(\mathrm{~nm})$ & $b(\mathrm{~nm})$ & $c(\mathrm{~nm})$ & $\gamma($ deg. $)$ & $\varepsilon_{\mathrm{T}}^{\mathrm{CBED}} \times 10^{-3}$ & $\varepsilon_{\mathrm{T}}^{\mathrm{T}} \times 10^{-3}$ & $\varepsilon_{\mathrm{T}}^{\mathrm{DCD}} \times 10^{-3}$ & $\varepsilon^{\prime} \times 10^{-3}$ \\
\hline P1 & 0.5429 & 0.5429 & 0.5429 & 90 & - & - & - & - \\
\hline P2 & $>0.5429$ & $>0.5429$ & $\geq 0.5433$ & $<90$ & $\geq 0.7$ & - & $\sim 1$ & $\leq 1$ \\
\hline P3 & 0.5427 & 0.5427 & 0.5418 & 90.04 & $(-2.0 \pm 0.4)$ & $(-2.5 \pm 0.3)$ & $(-2.7 \pm 0.1)$ & -1.9 \\
\hline
\end{tabular}

that occurs in TEM samples, thinned perpendicular to the direction of lattice parameter variation [20]. However, the agreement with DCD is reasonably good if we assume, as a first approximation, that the strain in the thinned specimens has the isotropic "fully relaxed" value $\varepsilon^{\prime}=(1+\nu) f$ (see Tab. I; here $\nu$ is assumed to be equal to 0.28 [21]). It must be pointed out that, if more accurate comparison between CBED and DCD measurements are needed, the strain value along the $\langle 001\rangle$ direction in $\langle 110\rangle$ cross-sectioned samples should be calculated according to the anisotropic elasticity theory. Nevertheless the first order isotropic results reported in table I, seems reasonable to us because of the experimental error which affects our strain measurements.

The difficulties caused by surface relaxation to strain quantification in cross-sectioned samples can be overcome by using the Bragg contour method in plan-view specimens, where relaxation phenomena are smaller.

Figure 5 gives a schematic view of the experimental arrangement adopted. Moving away from [001], the [012] zone axis is reached where planes $(0 \overline{8} 4)$ (or $(08 \overline{4})$ ) are in the Bragg position. When both the silicon and the implanted layers are illuminated by the electron beam, splitting is observed in the Bragg contours and the tilt angle can be measured. Subsidiary lines generated in the $\mathrm{Si}(\mathrm{B})$ layer appear distant from the [001] zone axis with respect to the perfect silicon ones. Note that the determination of the sign of the strain is possible without direct indexing of the diffraction pattern. According to equation (1), the (400) and (800) Bragg contours are unsplit and can be used for precise camera length measurements.

Figure 6 shows a direct beam LACBED [012] zone axis pattern of the plan-view specimen. It is clearly visible that the $(0 \overline{8} 4)$ and the $(2 \overline{4} 2)$ Bragg contours are split, whereas the $(800)$ contours are unsplit. From this LACBED pattern it is possible to deduce the angular shift between the surface $\mathrm{Si}(\mathrm{B})$ layer and the silicon substrate. The relative strain, calculated according to equation (1), is reported in table I. The agreement with X-ray DCD is reasonably good despite the fact that a small relaxation probably occurs even in the plan thinned specimen. From this strain value, the density of the substitutional boron atoms, $N_{\mathrm{i}}$, can be deduced from Vegard's law:

$$
N_{\mathrm{i}}=(1-\nu) \varepsilon_{\mathrm{T}} /(1+\nu) K
$$

with $K=-5.2 \times 10^{-24} \mathrm{~cm}^{3}$, as reported in literature [6]. Here $N_{\mathrm{i}}=2.7 \times 10^{20} \mathrm{~cm}^{-3}$ is in reasonable agreement with an electrical determination of the carrier concentration which gave $3 \times 10^{20} \mathrm{~cm}^{-3}[22]$.

It was found very difficult, however, to use this method to separate the contribution of the tensile strain from that of the compressive one induced by interstitial defects. This difficulty probably arises from the very small thickness of the defective layer (less than $10 \%$ of the diffracting volume). 


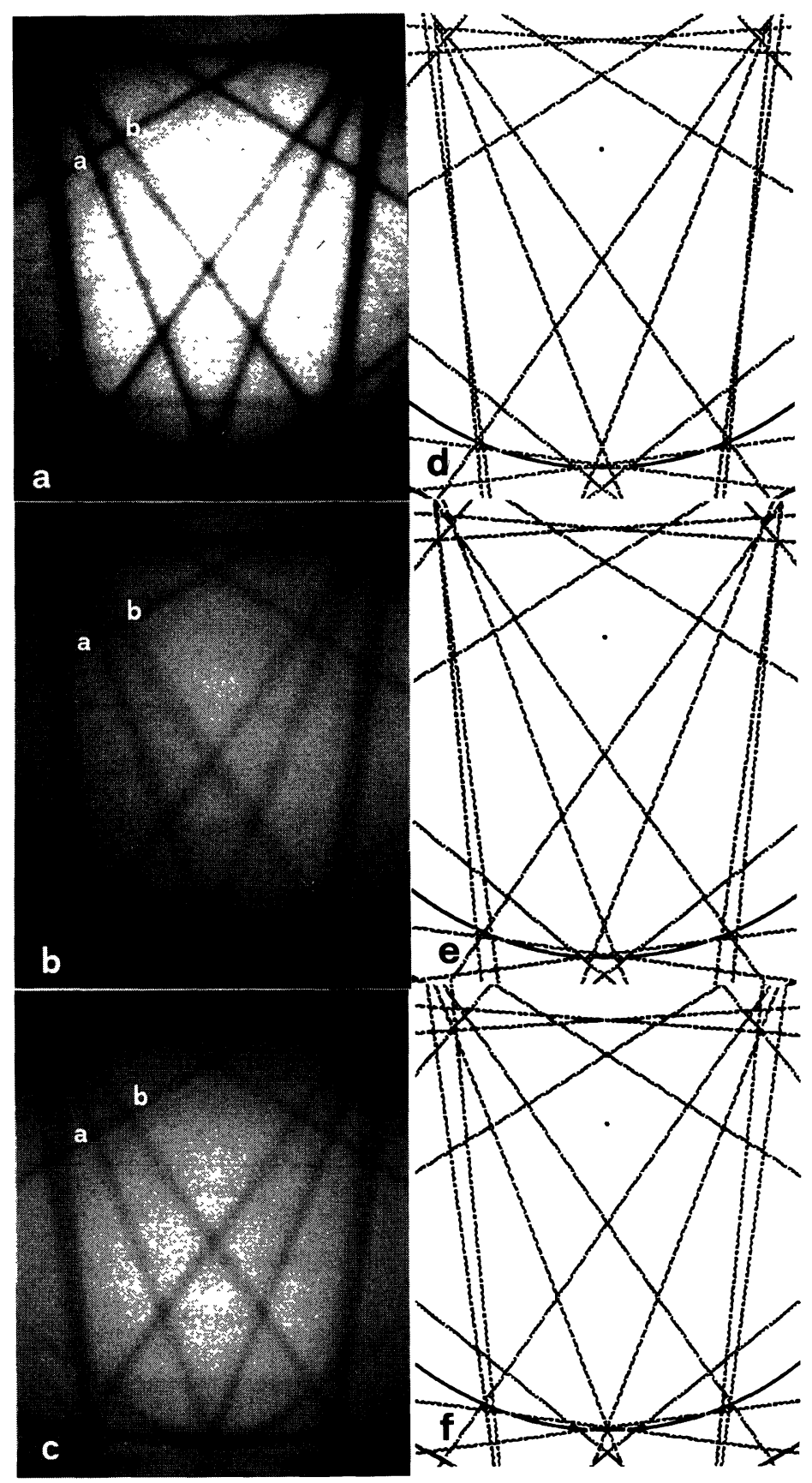

Fig. 4. - Experimental and calculated [310] CBED patterns taken at point P1 (reference state: a,d), P2 (compressive strain: b,e) and P3 (tensile strain: c,f) (see Fig. 2). The acceleration voltage fitting parameter is $99.2 \mathrm{kV}$. The corresponding strain values are reported in table $\mathrm{I}$. 

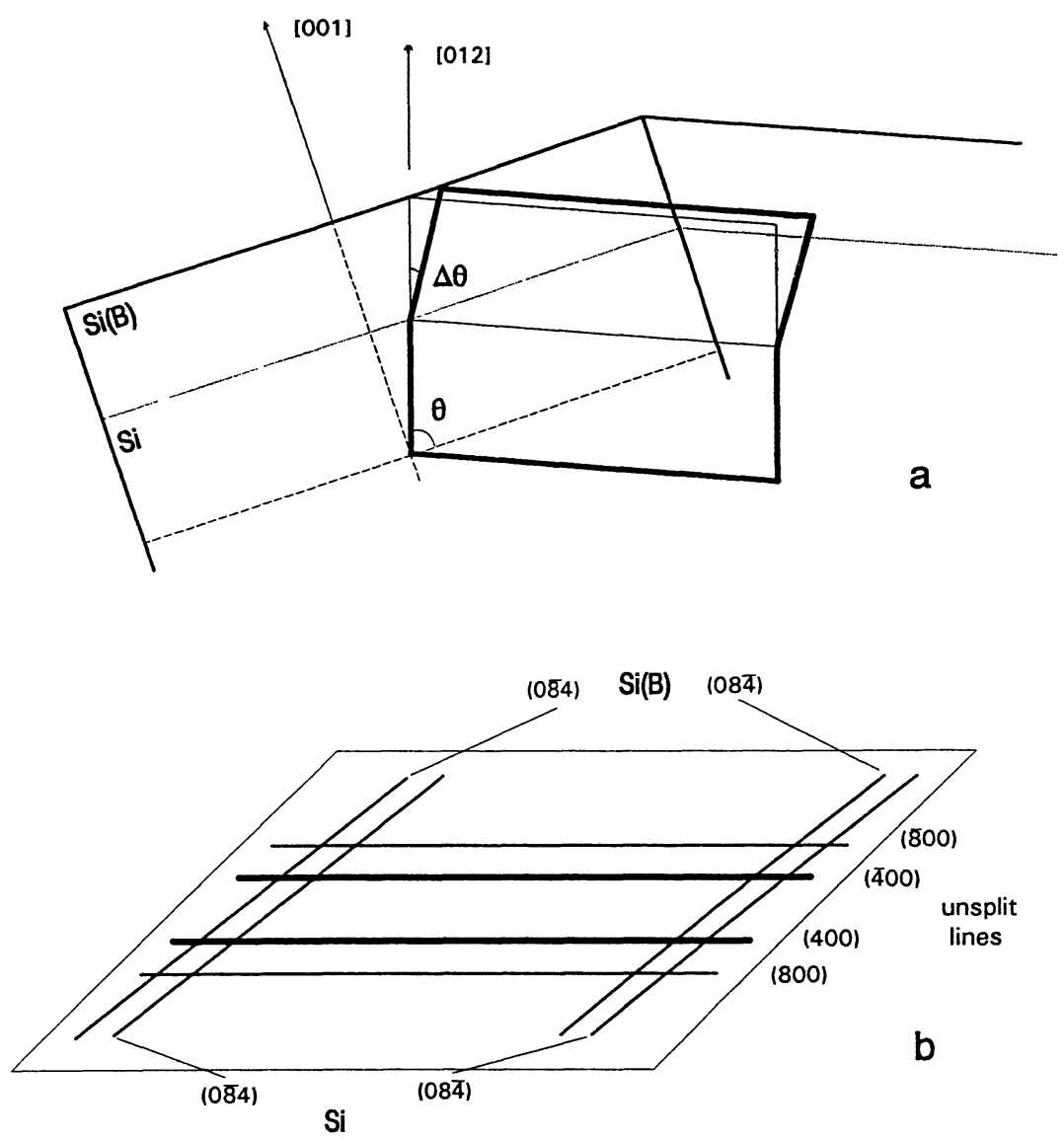

Fig. 5. - a) Experimental arrangement adopted to obtain LACBED patterns (see Fig. 1). In b) a rough scheme of the observed diffraction pattern is shown.

\section{Conclusions.}

Our investigation of the application of CBED technique to the study of strains in boron implanted silicon layers provided results in agreement with bulk data in plan thinned specimens and with the fully relaxed strain values in [110] cross-sectioned specimens. Our strain measurements allowed us to estimate a concentration of dopant atoms in substitutional sites in the $\mathrm{Si}(\mathrm{B})$ layer which is in agreement with electrical measurements. In cross-sectioned specimens a profile of the sign of the strain was made, revealing a difference between the silicon doped layer and the interstitial defective one. Work is in progress in order to apply the CBED technique to the study of the graded strain profile in germanium implanted silicon specimens. 


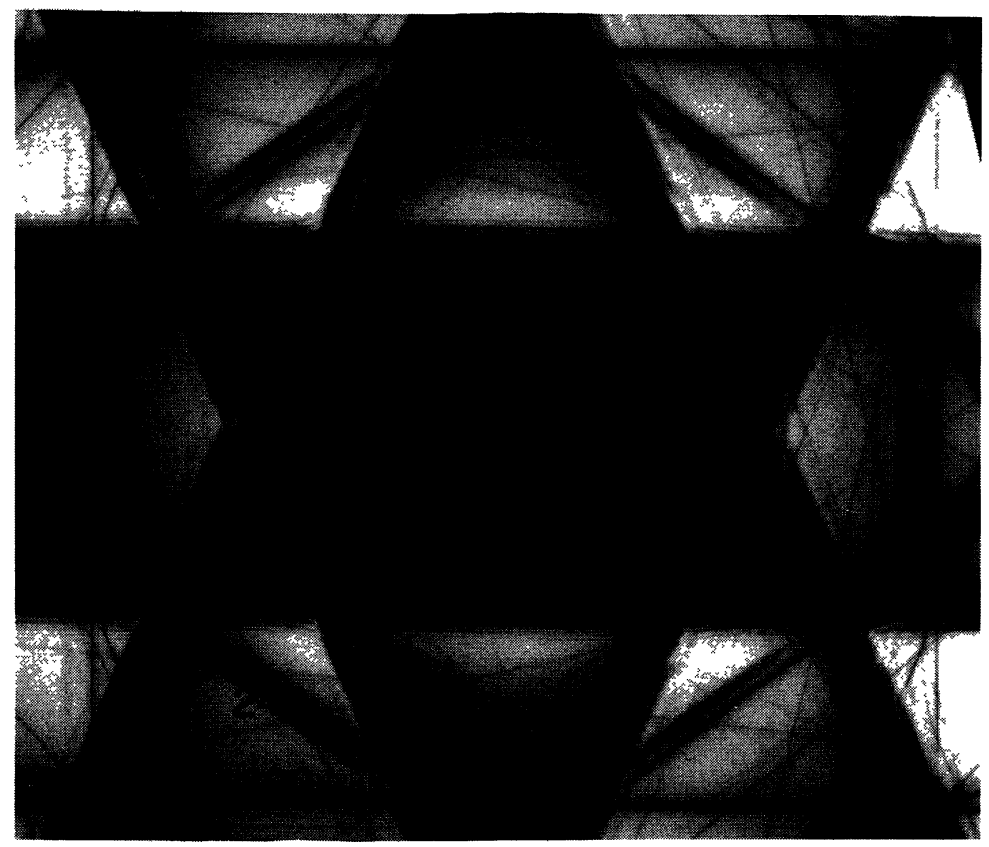

Fig. 6. - [012] LACBED pattern taken at $300 \mathrm{kV}$. The splitting of $(0 \overline{8} 4)$ and $(2 \overline{4} 2)$ Bragg contours is evident. The (400) and (800) Bragg contours are unsplit.

\section{Acknowledgements.}

The authors are indebted to: CNR-LAMEL Institute for use of the Philips CM30 TEM, M. Servidori (LAMEL Institute) for DCD measurements, G. Queirolo (ST-Microelectronics) for electrical measurements, $R$. Tonini (Dipartimento di Fisica, Universita' di Modena) for ion implantation and annealing of the samples, A. Armigliato (LAMEL Institute) and G. Ottaviani (Dipartimento di Fisica, Universita' di Modena) for stimulating discussion. This work has been supported by Ministero dell'Universita'e Ricerca Scientifica e Tecnologica (M.U.R.S.T.).

\section{References}

[1] Jones P.M., RACKHAM G.M. and STEEDS J.W., Proc. R. Soc. Lond. A 354 (1977) 197.

[2] Maher D.M., Fraser H.L., Humphreys C.J., KnOell R.V. and Bean J.C., Appl. Phys. Lett. 50 (1987) 574.

[3] TwIG M.E. and CHU S.N.G., Ultramicroscopy 26 (1988) 51.

[4] DUA X.F., Fung K.K., CHU Y.M., Sheng C. and ZHOU G.L., Philos. Mag. 63 (1991) 79.

[5] Rossouw C.J., AL-KHAFAII M., CHERNS D., STEEDS J.W. and TOUAITIA R., Ultramicroscopy 35 (1991) 229.

[G CARdona M., Christensen N.E., Phys. Rev. B 35 (1987) 6182.

[7] HORNSTRA J. and BARTELS W.J., J. Crystal Growth 44 (1978) 513.

[8] LARSON B.C., J. Appl. Phys. 45 (1974) 514.

[9] SERVIDORI M., unpublished results. 
[10] Garulli A., ARMigliato A. and VANZi M., J. Microsc. Spectrosc. Electron. 10 (1985) 135.

[11] SERVIDORI M., Nucl. Instrum. Meth. Phys. Res. B 19/20 (1987) 443.

[12] HUMPHREYS C.J. and EAGLESHAM D.J., MAHER D.M. and FRASER H.L., Ultramicroscopy 26 (1988) 13.

[13] CHERns D. and PREsTon A.R., J. Electron Microsc. Tech. 13 (1989) 111.

[14] Lin Y.P., PREston A.R., VinCENT R., I.o.P. Conf. Ser. (I.o.P. Publishing, Bristol) 90 (1987) 115.

[15] BITHELL E.G. and STOBBS W.M., J.Microsc. 153 (1989) 39.

[16] Landi E., Guimaraes S. and Solmi S., Appl. Phys. $A 44$ (1987) 135.

[17] TaNaKa M., SaITO R., UENo K. and HaRADA Y., J. Electron Microsc. 29 (1980) 412.

[18] Ottaviani G., Nava F., Tonini R., Frabboni S., Cerofolini G.F. and CanToni P., Mat. Res. Soc. Symp. Proc. 147 (1989) 85.

[19] STADELMANN P.A., Ultramicroscopy 21 (1987) 131.

[20] TREACY M.M.J. and GIBSON J.M., J. Vac. Sci. Technol. B 4 (1986) 1458.

[21] BRantley W.A., J. Appl. Phys. 44 (1973) 534.

[22] QUEIROLO G., unpublished results. 